$\mathrm{N}$ o $\mathrm{t}$ e.

Settembre 22 a 25 . Il nucleo è rotondo, planetario, grande circa come Giove $\left(37^{\prime \prime}\right)$; per esso passa la chioma in forma di largo e lungo arco parabolico, colla massima larghezza sul nucleo medesimo, seguita da uno spazio oscuro.

Settembre 26 a 28 . Con ingrandimenti di 210 e 340 si vede il contorno del nucleo meno deciso e con una prominenza diretta verso il sole. La chioma forma un arco o falce più stritta, passante per il nucleo.

Settembre 30 a Ottobre 2. Nucleo doppio: la massa luminosa più vicina al sole è diffusa verso $S E$, e più larga dell altra, e ne è divisa da una strozzatura meno lucida, la retta passante per i due nuclei (dal precedente al seguente nel moto diurno) fa un angolo di circa $10^{\circ}$ coll' asse della cometa presso il nucleo. L'oscurità nell' interno della chioma diviene sempre meno distinta.

Ottobre 3 a 7 . La massa nucleare più vicina al sole $\mathrm{e}$ più larga e più lunga dell' altra e contiene un punto molto vivo; la chioma forma un arco debole e breve; l'oscurità interna scompare.
Ottobre 8 a Novembre. 23. Ise due masse nucleari si alJungano sempre più; al Ottobre 20 il passaggio dura I:3 (lunghezza circa 20"); poi divengono sempre più diffuse, ed è difficile al riconoscerne la vera figura: sembrano entrambe piriformi, e costituiscono un complesso a forma di rombo allungatissimo.

Novembre 24 a Febbr. 23. Il nucleo è fusiforme, sempre più allungato, alquanto più lucido nell' asse; in esso con ingrandimenti da 90 a $140 \mathrm{si}$ riconoscono $\mathrm{par}$ ecchi punti più vivi, il numero dei quali è spesso incerto, e pare diverso nelle diverse osservazioni: Novembre 24 un massimo di luce nella parte occidentale; Gennajo 9 un punto vivo nel mezzo; Gennajo 3 tre o quatro punti lucidi; Gennajo 8 due o tre, Gennajo 29 tre; Gennajo 30 due; Febbrajo 5 due; Febbrajo 6 due; Febbrajo 23 due.

Febbrajo 23. Luna all' orizzonte. 'Il nucleo è estremamente diffusa e debole: impossibili le misure micrometriche; la lunghezza è stimata di circa $3^{\prime}$.

\title{
Ephemeris of the Great Comet 1882 II.
}

[Communicated by Vice-Admiral Rozean Superintendent U. S. Naval Observatory.] Computed from elements (A. N. 2482 and 2490 ) and reduced to the mean Equinox 1883.0 Greenwich mean noon.

\begin{tabular}{|c|c|c|c|c|c|c|}
\hline \multicolumn{2}{|c|}{1883} & \multicolumn{2}{|r|}{ RA. } & Decl. & $\log r$ & $\log \Delta$ \\
\hline \multicolumn{2}{|c|}{ Febr. 10.0} & \multicolumn{2}{|c|}{$6^{\mathrm{h}} \quad 0^{\mathrm{m}} 37^{\mathrm{s}} .8$} & - I $9^{\circ} 4 I^{\prime} I 7^{\prime \prime}$ & \multirow{2}{*}{$\begin{array}{l}0.48 \text { I } 37 \\
0.48009\end{array}$} & $0.3^{88} 9 \mathrm{I}$ \\
\hline & 14.0 & 5 & 5740.4 & $1840 \quad 13$ & & 0.40520 \\
\hline & 18.0 & 5 & $\begin{array}{ll}55 & 19.7\end{array}$ & 174117 & 0.49669 & $0.4213^{2}$ \\
\hline & 22.0 & 5 & $53 \quad 32 \cdot 7$ & I6 4435 & 0.50413 & 0.43723 \\
\hline & 26.0 & 5 & $52 \quad 14.7$ & I $5 \quad 50 \quad 14$ & 0.5 I 133 & $0.45^{282}$ \\
\hline Mar. & 2.0 & 5 & $5^{1} \quad 24.4$ & $145^{8} 16$ & $0.5184 \mathrm{I}$ & 0.468 I 7 \\
\hline & 6.0 & 5 & $50 \quad 58.7$ & I $4 \quad 843$ & 0.52532 & 0.48322 \\
\hline & 10.0 & 5 & $50 \quad 54.8$ & $\begin{array}{lll}3 & 21 & 37\end{array}$ & 0.53200 & 0.49790 \\
\hline & 14.0 & 5 & $51 \quad 12.3$ & I 370 & $0.53^{861}$ & $0.5 \mathrm{I} 23 \mathrm{I}$ \\
\hline & 18.0 & & $51 \quad 47.9$ & I I $54 \quad 52$ & 0.54508 & 0.52635 \\
\hline & 22.0 & 5 & $5^{2} \quad 39.5$ & II 15 IO & 0.55135 & 0.53995 \\
\hline & 26.0 & 5 & 5346.1 & I0 $375^{6}$ & 0.55751 & 0.55316 \\
\hline & 30.0 & 5 & $55 \quad 6.1$ & 10 36 & 0.56354 & $0.5^{6} 594$ \\
\hline Apr & 3.0 & 5 & $5^{6} 3^{8.1}$ & $930 \quad 34$ & 0.56944 & 0.57828 \\
\hline & 7.0 & 5 & 5820.9 & $9 \circ 19$ & 0.57520 & 0.59015 \\
\hline & I I.O & 6 & - 13.9 & $-83^{2} 21$ & 0.58090 & $0.6015^{8}$ \\
\hline
\end{tabular}

Washington I $883_{3}$ Febr. Io.

E. Frisby, Prof. math., U. S. N.

Obgleich eine Ephemeride des Hern Stechert nach den Elementen von Dr. Kreutz bereits vorliegt, die noch nahe mit dem Himmel stimmt, glaubte ich doch, dass die obige auch von Interesse für die Leser sein könnte. 